\title{
Analysis of the causes and effectiveness of whole blood transfusion procedures carried out in dogs and cats in 2014-2018 in a Polish veterinary clinic
}

\author{
JUSTYNA WOJTAŚ, ALEKSANDRA GARBIEC \\ Department of Ethology and Wildlife Management, Faculty of Animal Sciences and Bioeconomy, \\ University of Life Science in Lublin, Akademicka 13, 20-950 Lublin, Poland
}

Wojtaś J., Garbiec A.

Analysis of the causes and effectiveness of blood transfusion procedures carried out in dogs and cats in 2014-2018 in a Polish veterinary clinic

Summary

Nowadays, veterinary medicine develops as quickly as human medicine, and companion animals are treated as family members. As a result, advanced procedures, such as blood transfusions, are used more frequently to save animal life. The present study analyzes blood transfusions carried out in dogs and cats in 2014-2018 in a veterinary clinic for small animals in Poland. The number of transfusions performed, indications for their implementation due to disease, as well as the effects of this procedure, are discussed. This method of treatment, although potentially life-saving, is not always effective and carries a risk of adverse reactions.

Keywords: dogs, cats, transfusion, diseases

According to data published by the European Pet Food Industry Federation in 2017, there were over 84 million dogs living in Europe at that time. An estimated 80 million European households own at least one pet animal, and in every fifth household that animal is a dog (http://www.fediaf.org/who-we-are/ european-statistics.html). Dogs are becoming increasingly important as family members rather than merely companion animals. In cases of sudden diseases and life-threatening conditions, the owners want to save their pets at any cost. Veterinary medicine is constantly developing and applying life-saving methods, such as those used in human medicine. An example of such a medical procedure is a whole blood transfusion.

The very first successful direct blood transfusion between animals was carried out in 1665 by the London anatomist Richard Lower. What Lower did was to transfuse blood from the carotid artery of one dog to the jugular vein of the other (13). Years of research, scientific development and technological progress have made blood transfusions an integral part of the treatment of patients with anemia. Blood transfusion is a potentially life-saving procedure for both humans and animals (20). Blood and blood products are already readily available in veterinary practice, and transfu- sions can be performed in many clinics. However, it is vital to know the potential risks associated with blood transfusion, which, although rare, may be lifethreatening. Safe blood transfusions and safe use of blood products require basic knowledge about blood groups and the awareness of the presence of antibodies. Correct selection of appropriate donors and screening tests is also necessary (12).

Blood groups occur in both dogs and cats. There are 13 blood groups in dogs, but only 8 are commonly recognized. In the case of cats, 3 different blood groups are distinguished. The groups have been distinguished on the basis of antigens present on the surface of erythrocytes (16). Blood groups in dogs are distinguished by dog erythrocyte antigens (DEA): $1(1.1,1.2,1.3)$ ), $3,4,5,6,7,8$. To avoid undesirable post-transfusion reactions, it is necessary to determine blood groups or perform a cross-match test before blood transfusion. The DEA 1.1 and 1.2 antigens are the most important, as they are present in approximately $60 \%$ of dogs and have the highest serum titer. After DEA 1.1 blood transfusion into DEA 1.2 dogs, anti-DEA 1.1 antibodies are produced, which is the most common cause of an adverse post-transfusion reaction (19). The DEA 4 antigen is found in up to $98 \%$ of dogs, therefore dogs 
with this antigen are considered to be the best donors due to the low risk of hemolytic transfusion reactions (5). The blood of the DEA 1 group gives the strongest antigen response and may lead to an immunologic reaction during the next transfusion, which is why it is commonly believed that the first blood transfusion carries a small risk, but the risk increases significantly with each subsequent transfusion. To avoid adverse post-transfusion reactions in dogs, the DEA 1.1 group should be confirmed or ruled out before blood transfusion by blood group determination. Cross-matching is also recommended to exclude the phenomenon of agglutination of blood cells. As already mentioned, cats have three different blood groups: A, B, AB (6). Blood group A is present in about $90 \%$ of cats (8). Unlike dogs, cats have naturally occurring antibodies with quite significant titers, which is a major obstacle to safe transfusions (2). Due to the high risk of blood transfusion in cats, it is performed extremely rarely, following a thorough laboratory analysis of the blood of the recipient and the donor. Blood groups are determined by taking a whole blood sample into an EDTA tube from both the donor and the recipient. In emergencies, the most common method of blood selection for transfusion is cross-matching the donor's and the recipient's blood samples.

Dog blood donors are selected for their age, weight, health status, past diseases, regularity of prophylaxis, and even temperament. The ideal donor is a healthy young male or female, weighing over $30 \mathrm{~kg}$, regularly vaccinated and dewormed, with ideal results of morphological and biochemical blood tests performed at least once a year (12). A dog can become a donor only if it has not received a blood transfusion. In the case of cats, the donor can be an individual with a body weight of not less than $4 \mathrm{~kg}$, free from infectious diseases and regularly dewormed. Every 6 weeks, $15 \mathrm{ml}$ of blood per $\mathrm{kg}$ of body weight can be taken from a dog and 10-12 ml per kg body weight from a cat. Cats should be sedated (11).

Although a blood transfusion is a life-saving procedure, it is quite risky. It can result in complications, the so-called adverse post-transfusion reactions. Their onset may be sudden and occur already during transfusion (acute hemolytic transfusion reactions) or with a delay, from 24 hours to as much as several days after treatment (delayed hemolytic transfusion reactions). The most frequent adverse reactions are non-hemolytic transfusion reactions (14). They are allergic reactions and fever (15). Adverse transfusion reactions are most common in dogs that have previously been sensitized to the DEA 1.1 antigen (past transfusion) and in cats that have been given non-compatible blood. The most common disturbing signs occurring immediately after the start of transfusion include fever, changes in the heart rate, hypotension, dyspnoea, vomiting, hemoglobinemia, and hemoglobinuria (3).

\section{Material and methods}

The data presented in the current article refer to blood transfusions carried out in 2014-2018 in a veterinary clinic for small animals in Poland. The blood recipients were dogs and cats that were patients of the clinic. During those 5 years, a total of 177 blood transfusions were performed. The most transfusions occurred in 2017. Among the animals that had their blood transfused, dogs vastly outnumbered cats (Fig. 1). Among the dogs qualified for a transfusion procedure, the majority suffered from a complicated course of tick-borne disease and coagulation disorders due to rodenticide poisoning. In cats, the main indications for blood transfusion were IMHA and complicated viral diseases (Tab. 1, Tab. 2). The animals were qualified for transfusion on the basis of a poor clinical condition associated with deteriorating morphological findings. All transfusion recipients were tested for their blood type by the RapidVet blood test and a cross-check. The donors had a routine blood type test. During transfusion, the patient received 12-20 ml of whole blood $/ \mathrm{kg}$ of body weight at a rate of 3-4 ml/ $\mathrm{kg}$ of body weight/hour. The entire procedure never took more than 4 hours (18). There is a veterinary blood bank in the

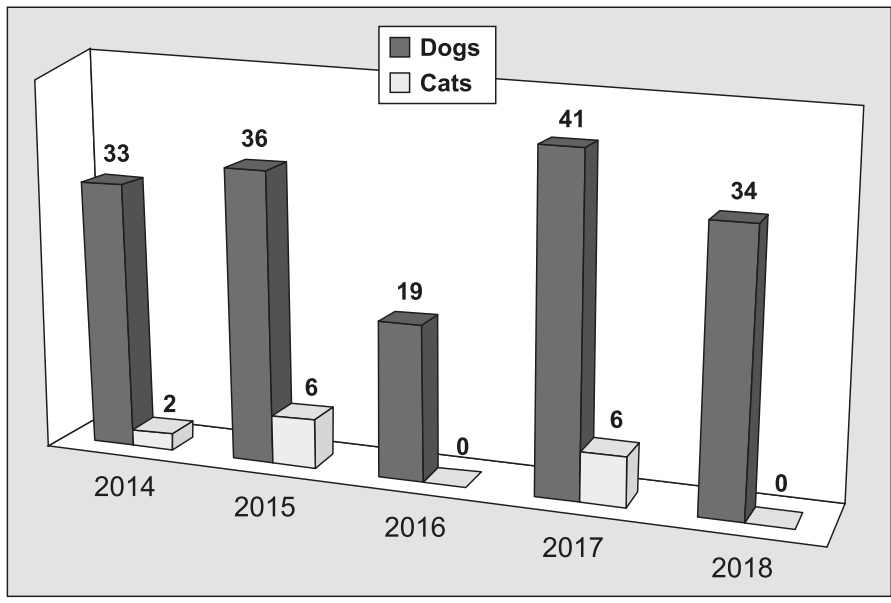

Fig. 1. Blood transfusions in dogs and cats (2014-2018)

Tab. 1. Complicated basic diseases that were indications for transfusion in dogs (2014-2018)

\begin{tabular}{|l|c|c|c|}
\hline \multicolumn{1}{|c|}{ Diseases } & Successful & Unsuccessful & All \\
\hline Babesiosis & 48 & 15 & 63 \\
Rodenticide intoxication & 23 & 6 & 29 \\
\hline Ruptured spleen tumor & 5 & 8 & 13 \\
Cancer process & 1 & 11 & 12 \\
\hline Kidney failure & 5 & 4 & 9 \\
IMHA & 5 & 3 & 8 \\
Bloody surgery & 2 & 5 & 7 \\
Parasitosis & 3 & 2 & 5 \\
\hline Pyometra & 2 & 2 & 4 \\
Gastroenteritis & 1 & 3 & 4 \\
Trauma & 1 & 3 & 4 \\
Pancreatitis & 1 & 2 & 3 \\
\hline Anaplasmosis & 1 & 0 & 1 \\
Cushing syndrome & 0 & 1 & 1 \\
\hline
\end{tabular}


Tab. 2. Complicated basic diseases that were indications for transfusion in cats (2014-2018)

\begin{tabular}{|l|c|c|c|}
\hline \multicolumn{1}{|c|}{ Diseases } & Successful & Unsuccessful & All \\
\hline IMHA & 1 & 3 & 4 \\
\hline Leukemia & 0 & 3 & 3 \\
\hline Rodenticide intoxication & 1 & 1 & 2 \\
Cancer process & 0 & 2 & 2 \\
\hline Parasitosis & 1 & 0 & 1 \\
Pyometra & 1 & 0 & 1 \\
\hline Pancreatitis & 0 & 1 & 1 \\
\hline
\end{tabular}

clinic where the statistical tests were carried out. The study analyses the transfusions in terms of their efficacy and indications, in both dogs and cats.

\section{Results and discussion}

The whole blood transfusions discussed here were 177 life-saving treatments performed in 163 dogs and 14 cats. The effectiveness of all was 57\%. Out of 163 blood transfusions performed in dogs, 98 were successful and produced the desired effect (60\% efficacy). In cats, only 4 out of 14 transfusions were successful (29\% efficacy) (Fig. 2). Successful transfusions were those that contributed to the recovery of animals, e.g. by improving red cell parameters, shortening blood clotting time, and correcting hypovolemia. In the remaining animals, transfusions were considered unsuccessful because they failed or were not completed due to a post-transfusion reaction or cardiopulmonary failure/arrest or the patient's death despite resuscitation. The effectiveness of full blood transfusions in dogs was almost twice as high as in cats, which may be due to the presence of naturally occurring antibodies with significant titers in cats (7). Even with the right choice of blood group, the cat's immune system can still produce antibodies that react with the blood of the donor (18). This is likely to cause non-hemolytic reactions, including acute lung injury with increasing

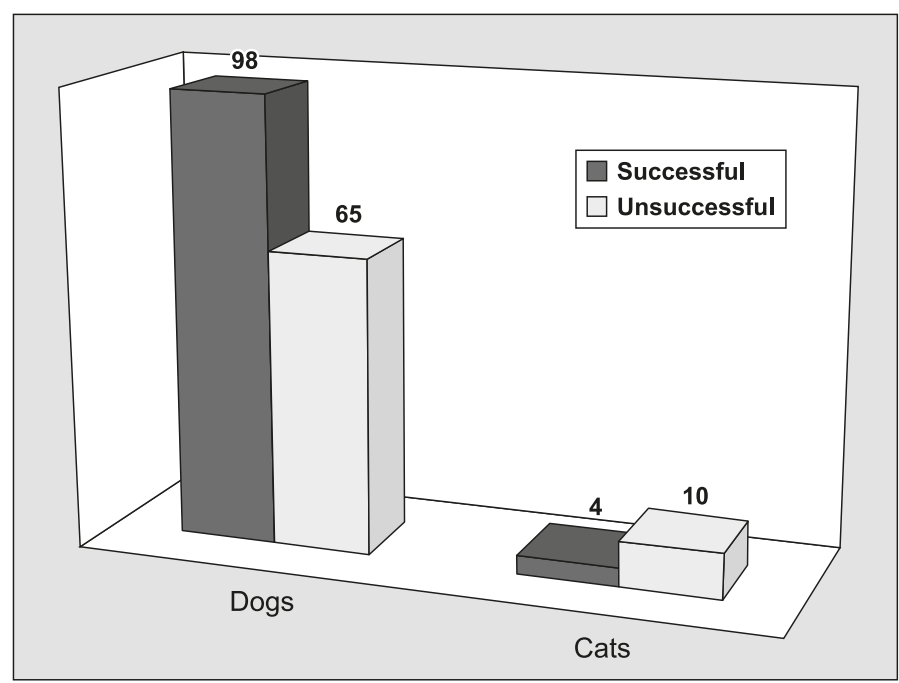

Fig. 2. The effectiveness of blood transfusions in dogs and cats (2014-2018) dyspnea, tachypnea, and fever (1). Delayed reactions are the most common reactions after group-compatible blood transfusion in cats. One of their characteristics is a shortened survival time of transfused erythrocytes (9). Moreover, in a significantly weakened cat in poor condition, even mild and temporary post-transfusion reactions often lead to failure of the procedure. Adverse reactions may be due to serious difficulties in storing feline blood, mismatched amounts of blood administered, or blood contamination (10).

The basic indication for a blood transfusion is hematocrit (anemia) with a threshold of $15 \%$ (4). The main causes of anemia include massive hemorrhages, coagulopathies (e.g. in rodenticide poisoning), surgical procedures that carry a risk of extensive blood loss, haemophilia, disseminated intravascular coagulation syndrome (DIC) $(\mathrm{n}=12)$, breakdown of red blood cells (e.g. in babesiosis), infectious diseases (e.g. FeLV, canine distemper), renal failure, and neoplastic processes $(n=16)$. In the current study, the primary causes of blood transfusion in dogs were babesiosis $(\mathrm{n}=63)$, rodenticide intoxication $(\mathrm{n}=29)$, ruptured spleen tumor $(n=13)$, cancer process $(n=12)$, kidney failure $(n=9)$, IMHA $(n=8)$, severe blood loss following a surgery $(n=7)$, parasitic infestation $(n=5)$, pyometra $(n=4)$, gastroenteritis $(n=4)$, trauma $(n=4)$, pancreatitis $(\mathrm{n}=3)$, anaplasmosis $(\mathrm{n}=1)$, and Cushing syndrome $(\mathrm{n}=1)(\mathrm{Tab} .1)$. With regard to cats, the causes were IMHA (immune-mediated hemolytic anemia) $(n=4)$, leukemia $(F e L V),(n=3)$, rodenticide intoxication $(n=2)$, cancer process $(n=2)$, parasitic infestation $(n=1)$, pyometra $(n=1)$, and pancreatitis $(n=1)$ (Tab. 2). Transfusions in dogs with babesiosis with advanced breakdown of red blood cells (advanced anemia), hyperbilirubinemia, as well as renal and hepatic failure accounted for approximately $40 \%$ of all transfusions performed in 2014-2018. In 18\% of dogs, poor health was due to rodenticide poisoning. Nearly $1 / 3$ of cats received a transfusion because of IMHA. In all cases, transfusion was a life-saving procedure due to deteriorating health despite pharmacological treatment. Depending on the underlying disease, the animals received antiprotozoal and antiparasitic drugs, steroids, antibiotics, antihaemorrhagics, albumin, and infusion fluids. In animals undergoing life-saving surgery, the transfusion process began before surgery and continued throughout the surgery. All animals were under 24-hour medical care in the veterinary clinic.

Blood transfusion therapy is already an integral element of rescue medicine in small animals. The procedure is performed much more often in dogs than in cats because of the greater availability of donors and easier collection and storage of blood. In cats, it is still rarely performed. This is probably due to the lower availability of blood (lack of long-term storage), difficulties in finding appropriate donors, the need for general anesthesia to collect donor blood, and the possibility of numerous post-transfusion reactions. 


\section{References}

1. Auer L., Bell K.: Transfusion reactions in cats due to AB blood group incompatibility. Res. Vet. Sci. 1983, 35, 145-152.

2. Blais M. C., Berman L., Oakley D. A., et al.: Canine Dal bloodtype: a red cell antigen lacking in some Dalmatians. J. Vet. Intern. Med. 2007, 21, 281-286.

3. Brown D.: Principles of blood transfusion and crossmatching, [in:] Thrall M. A., Weiser G., Allison R. W. (eds): Veterinary hematology and clinical chemistry. Ames I. A.: Wiley-Blackwell 2012, 205-222.

4. Cotter S. M.: Clinical transfusion medicine. Adv. Vet. Sci. Comp. Med. 1991, 187-223.

5. Feldman B., Zinkel J. G., Jain N. C.: Schalm's Veterinary Hematology. Lippincott Williams and Wilkins 2000, 864.

6. Giger U., Bucheler J., Patterson D. F.: Frequency and inheritance of A and B blood types in feline breeds of the United States. J. Hered. 1991, 82, 15-20.

7. Griot-Wenk M. E., Giger U.: Feline transfusion medicine: blood types and their clinical importance. Vet. Clin. N Am.-Small. 1995, 25, 1305-1322.

8. Hale A. S.: Canine blood groups and their importance in veterinary transfusion medicine. Vet. Clin. North Am. Small Anim. Pract. 1995, 25, 1323-1332.

9. Hansen K.: Canine and Feline Transfusion Medicine. Veterinary Technician, March 2019

10. Kohn B., Weingart C.: Feline Blood Typing and Transfusion - A Practica Approach. World Small Animal Veterinary Association World Congress Proc. 2006.

11. Kumar R.: Blood transfusion in veterinary medicine. Hematol. Transfus. Int. J. 2017, 4, 116-122.
12. Lanevschi A., Wardrop J.: Principles of transfusion medicine in small animals. Can. Vet. J. 2001, 42, 447-454.

13. Lower R.: The success of the experiment of transfusing the blood of one animal into another. Philos Trans R Soc. Lond. B Biol. Sci. 1665, 1, 352.

14. Maglaras C. H., Koenig A., Bedard D. L., Brainard B. M.: Retrospective evaluation of the effect of red blood cell product age on occurrence of acute transfusion-related complications in dogs: 210 cases (2010-2012). Journal of Veterinary Emergency and Critical Care 2017, 27, 108-120.

15. Sink C.: Practical Transfusion Medicine for the Small Animal Practicioner. John Wiley \& Sons 2017, 49-52.

16. Symons M., Bell K.: Expansion of the canine A blood group system. Anim. Genet. 1991, 22, 227-235.

17. Tocci L. J.: Transfusion medicine in small animal practice. Vet. Clin. N. Am.Small. 2010, 40, 485-494.

18. Tocci L. J., Ewing P. J.: Increasing patient safety in veterinary transfusion medicine: an overview of pretransfusion testing. Journal of Veterinary Emergency and Critical Care 2009, 191, 66-73.

19. Vap L. M.: Aktualne doniesienia na temat oznaczania grup krwi, wykonywania prób krzyżowych i bezpiecznego przeprowadzania transfuzji u psów i kotów. Weterynaria po Dyplomie 2011, 12.

20.Zaremba R., Brooks A., Thomovsky E.: Transfusion Medicine: An Update on Antigens, Antibodies and Serologic Testing in Dogs and Cats. Topics in Companion An. Med. 2019, 34, 36-46.

Corresponding author: Aleksandra Garbiec, DVM, ul. Akademicka 13, 20-950 Lublin; e-mail: aleksandra.garbiec@up.lublin.pl 